\title{
Ultrastructure of the flagellar apparatus in cryptomorphic Cryptomonas curvata (Cryptophyceae) with an emphasis on taxonomic and phylogenetic implications
}

\author{
Seung Won Nam ${ }^{1,2}$ and Woongghi Shin ${ }^{1, *}$ \\ ${ }^{1}$ Department of Biology, Chungnam National University, Daejeon 34134, Korea \\ ${ }^{2}$ LOHABE, Department of Oceanography, Chonnam National University, Gwangju 61186, Korea
}

Cryptomonas curvata Ehrenberg is a photosynthetic freshwater flagellate and the type species of the genus Cryptomonas. We examined the flagellar apparatus of cryptomorphic C. curvata by transmission electron microscopy. The major components of the flagellar apparatus are the non-keeled rhizostyle (Rhs), striated fibrous root (SR), striated fiber-associated microtubular root (SRm), mitochondrion-associated lamella (ML), and two types of microtubular roots (3r and 2r). The non-keeled Rhs originate at the ventral basal body and consist of two types of microtubule bands extending together into the middle of the cell. The SR and SRm extend parallel to the left side of the cell. The ML originates from the ventral basal body and is a plate-like fibrous structure associated with mitochondria. The $3 \mathrm{r}$ extends from the dorsal basal body toward the dorsal anterior of the cell. The $2 \mathrm{r}$ originates between the two basal bodies and extends shortly to the left of the cell. The overall configuration of the flagellar apparatus is most similar to that previously reported for C. pyrenoidifera. These results demonstrate that the features of the flagellar apparatus are useful for distinguishing closely related species and inferring phylogenetic relationships among taxa.

Key Words: cryptomonad; Cryptomonas; cryptomorph; diagrammatic reconstruction; flagellar apparatus; rhizostyle; transmission electron microscopy; ultrastructure

Abbreviations: af, anchoring fiber; ITS, internal transcribed spacer; LSU rDNA, large subunit of rDNA; ML, mitochondrion-associated lamella; Rhs, rhizostyle; SR, striated fibrous root; SRm, striated fibrous root-associated microtubular root; TEM, transmission electron microscopy; TIF, tagged image file; 2 r, two-stranded microtubular root; $3 r$, three-stranded microtubular root

\section{INTRODUCTION}

Cryptomonas species inhabit freshwater and slightly brackish waters worldwide (Choi et al. 2013, Xia et al. 2013). Cryptomonas is the oldest cryptophycean genus and includes approximately 50 species, representing approximately $25 \%$ of the total number of reported species (Novarino 2003, Guiry and Guiry 2015). However, when Ehrenberg established the genus, he did not designate a type species for the genus Cryptomonas (Hoef-Emden and Melkonian 2003, Novarino 2003). Recently, HoefEmden and Melkonian (2003) designated Cryptomonas curvata, with two described morphological types (cryptomorph and campylomorph), as a type species because this species apparently refers to only one taxon and was clearly assigned to a phylogenetic clade.
(9) $\$$ This is an Open Access article distributed under the terms of the Creative Commons Attribution Non-Commercial License (http://creativecommons.org/licenses/by-nc/3.0/) which permits unrestricted non-commercial use, distribution, and reproduction in any medium, provided the original work is properly cited.
Received March 28, 2016, Accepted June 13, 2016

*Corresponding Author

E-mail: shinw@cnu.ac.kr

Tel: +82-42-821-6409, Fax: +82-42-822-9690 
In previous studies, a cryptophycean group with brown coloration was found to consist of two genera, Cryptomonas and Campylomonas, which both exhibit the accessory pigment phycoerythrin 566 (Hill and Rowan 1989, Hill 1991, Deane et al. 2002, Novarino 2003). These genera can also be recognized based on characteristics such as cell shape (absence vs. presence of a recurved posterior), the type of inner periplast components (oval plates vs. inner sheet) and the type of rhizostyle (non-keeled vs. keeled), among others (Hill 1991, Clay et al. 1999, Novarino 2003). However, several molecular studies have revealed that these two genera are polyphyletic (Marin et al. 1998, Deane et al. 2002, Hoef-Emden et al. 2002). This group was additionally shown to form a clade with Chilomonas, a colorless leucoplast-bearing cryptophycean genus. Therefore, Hoef-Emden and Melkonian (2003) revised these three genera as synonyms of Cryptomonas, and Campylomonas was defined as an alternate morph (campylomorph) of Cryptomonas.

The ultrastructures of the flagellar apparatuses of several cryptomonads have been described. The first ultrastructural study was performed by Mignot et al. (1968), who focused on the flagella apparatuses of the genera Cryptomonas, Goniomonas, and Rhodomonas. Flagellar apparatus data have been reported for seven species, including campylomorph of Cryptomonas paramecium (Roberts et al. 1981), cryptomorph of C. pyrenoidifera (Roberts 1984, Perasso et al. 1992), Hanusia phi and G. theta (Gillott and Gibbs 1983), Proteomonas sulcata (Hill and Wetherbee 1986), Rhinomonas reticulata var. atrorosea (Nam et al. 2013), and Goniomonas avonlea (Kim and Archibald 2013). In the genus Cryptomonas, the flagellar apparatus structures of two species have been described, including campylomorph of $C$. paramaecium (Roberts et al. 1981) and cryptomorph of C. pyrenoidifera (Roberts 1984, Perasso et al. 1992). These two species share some characteristics, such as a short periodicity of the striated fibrous root (SR) striped pattern, rhizostyle (Rhs) consisting of two components, a Cr root consisting of two microtubules, the existence of a mitochondrion-associated lamella (ML), and a short periodicity of the ML striations.

In this study, the ultrastructure of the flagellar apparatus in cryptomorphic C. curvata was described and compared with those of other cryptomonad species, particularly Cryptomonas species.

\section{MATERIALS AND METHODS}

\section{Sampling, culture, and species identification}

A clonal culture of C. curvata sajeom041611A was established by isolating a single cell from freshwater samples collected at Sajeom pond, Korea $\left(36^{\circ} 30^{\prime} 40^{\prime \prime} \mathrm{N}, 126^{\circ} 47^{\prime} 43^{\prime \prime}\right.$ E), on Apr 16, 2011. The culture was grown in $\mathrm{f} / 2$ medium in which all components were dissolved in distilled water and maintained at $20-22^{\circ} \mathrm{C}$ under conditions of a $14: 10$ light : dark cycle with $30 \mu \mathrm{mol}$ photons $\mathrm{m}^{-2} \mathrm{~s}^{-1}$ from cool white fluorescent tubes. To identify the strain, we used nuclear internal transcribed spacer (ITS) 2 and partial large subunit of rDNA sequences. Species identification followed the methods of Choi et al. (2013). The culture strain was deposited in the culture collection of Chungnam National University, Daejeon, Korea.

\section{Light microscopy}

Live cells of C. curvata were observed at 1,000× magnification using an Axio Imager A2 (Carl Zeiss Inc., Hallbergmoos, Germany) equipped with differential interference contrast optics. Light micrographs were acquired with an AxioCam HRc (Carl Zeiss Inc.) photomicrographic system attached to the microscope.

\section{Transmission electron microscopy}

For transmission electron microscopy (TEM), aliquots of the culture were pelleted by centrifugation for $2 \mathrm{~min}$ at $371 \times \mathrm{g}(5,000 \mathrm{rpm})$ in an Eppendorf centrifuge 5415D (Eppendorf, Hamburg, Germany). After removing the supernatant, the pelleted cells were fixed in $2.5 \%(\mathrm{~V} / \mathrm{V})$ glutaraldehyde mixed with $\mathrm{f} / 2$ culture medium for $1 \mathrm{~h}$ at $4^{\circ} \mathrm{C}$. The glutaraldehyde-fixed cell pellets were washed 3 times in $\mathrm{f} / 2$ culture medium and post-fixed in $1 \%(\mathrm{~W} / \mathrm{V})$ $\mathrm{OsO}_{4}$ for $1 \mathrm{~h}$ at $4^{\circ} \mathrm{C}$. Dehydration, embedding and polymerization were performed following the methods of Nam et al. (2012). The polymerized blocks were thin-sectioned at a thickness of $70 \mathrm{~nm}$. Serial sections were collected on formvar-coated slot copper grids, stained with $3 \%(\mathrm{w} / \mathrm{v})$ uranyl acetate and Reynold's lead citrate (Reynolds 1963) and examined and photographed with a JEM-1010 transmission electron microscope operating at $80 \mathrm{kV}$ (JEOL, Tokyo, Japan). Images of the sections were recorded on Kodak EM Film 4489 (Eastman Kodak Co., Rochester, NY, USA) and scanned to tagged image file (TIF) format using Epson Perfection V700 Photo (Epson Korea Co., Ltd., Seoul, Korea). Three-dimensional reconstructions were generated by Catia V5R16 (Dassault-Aviation, Argenteuil, France). 

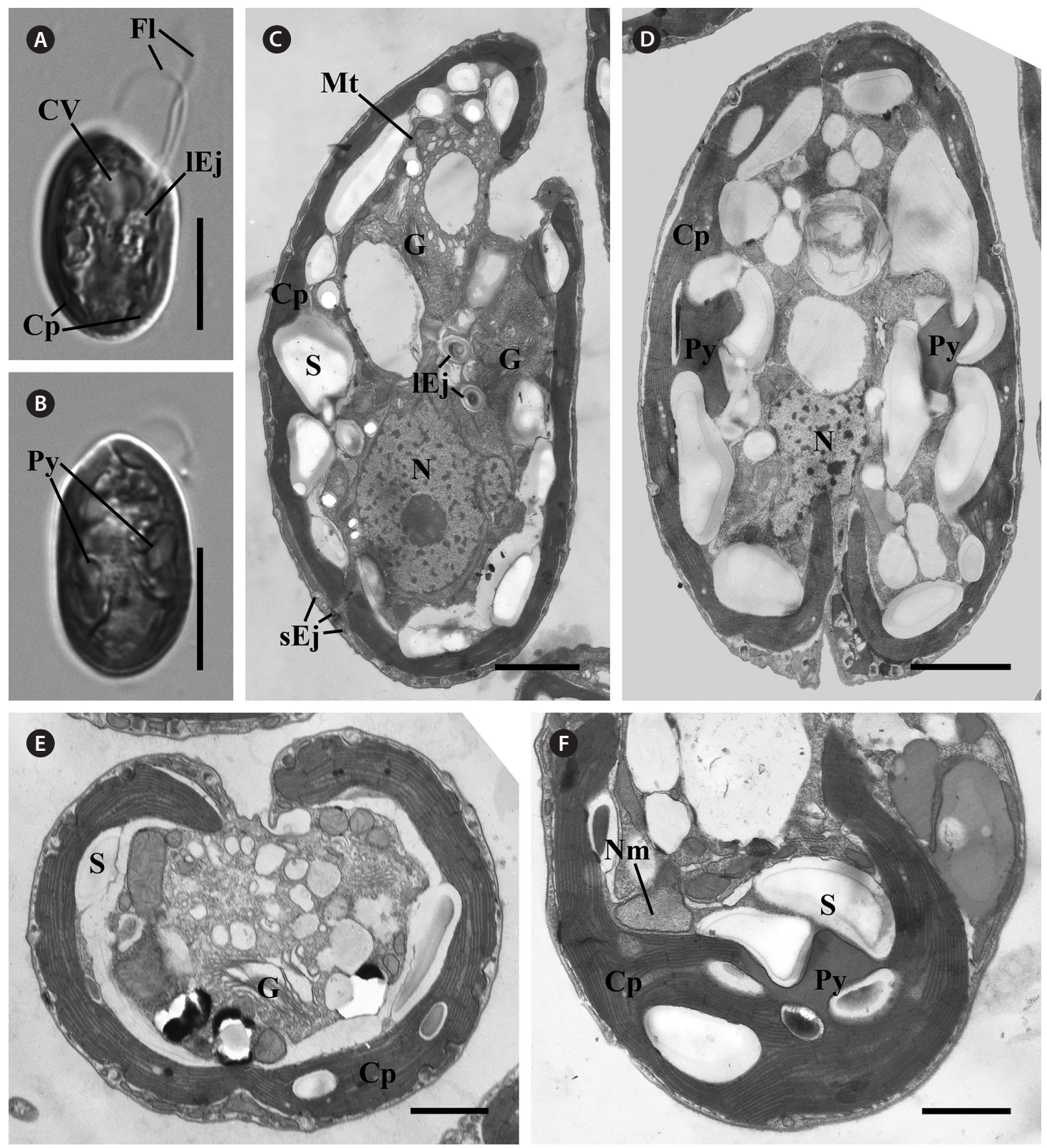

Fig. 1. Light and transmission electron micrographs of Cryptomonas curvata Sajeom041611A. (A) Light micrograph of the lateral view showing the contractile vacuole (CV), two flagella (FI), the large ejectosomes (IEj), and a chloroplast (Cp). (B) Light micrograph of the ventral view showing the brown chloroplast with two pyrenoids (Py). (C) Longitudinal section showing the peripheral chloroplast (Cp), Golgi bodies (G), nucleus (N), starch (S), mitochondria (Mt), and ejectisomes. Large ejectisomes (IEj) were located near the furrow / gullet system, and small ejectisomes (sEj) were located at the cell periphery. (D) Oblique section showing the chloroplast with two pyrenoids. (E) Cross section showing the one chloroplast, starch, and Golgi bodies (G). (F) Section of the periplastidial compartment showing the nucleomorph (Nm), which was not associated with the pyrenoid. Scale bars represent: $A \& B, 10 \mu \mathrm{m} ; C \& D, 2 \mu \mathrm{m} ; E \& F, 1 \mu \mathrm{m}$.

\section{RESULTS}

\section{Light microscopy}

C. curvata Sajeom041611A exhibited a dorsal convex shape in the lateral view (Fig. 1A) and an ovoid shape in the ventral view (Fig. 1B). The cells were 16.62-26.37 $\mu \mathrm{m}$ $(\mathrm{n}=57$ ) in length (mean, 20.46 \pm 2.24 ) and 10.72-15.34 $\mu \mathrm{m}$ $(\mathrm{n}=42)$ in width (mean, $12.39 \pm 1.05)$. The cells had two flagella and a brown chloroplast with two pyrenoids. A contractile vacuole was observed in the anterior portion (Fig. 1A \& B). 

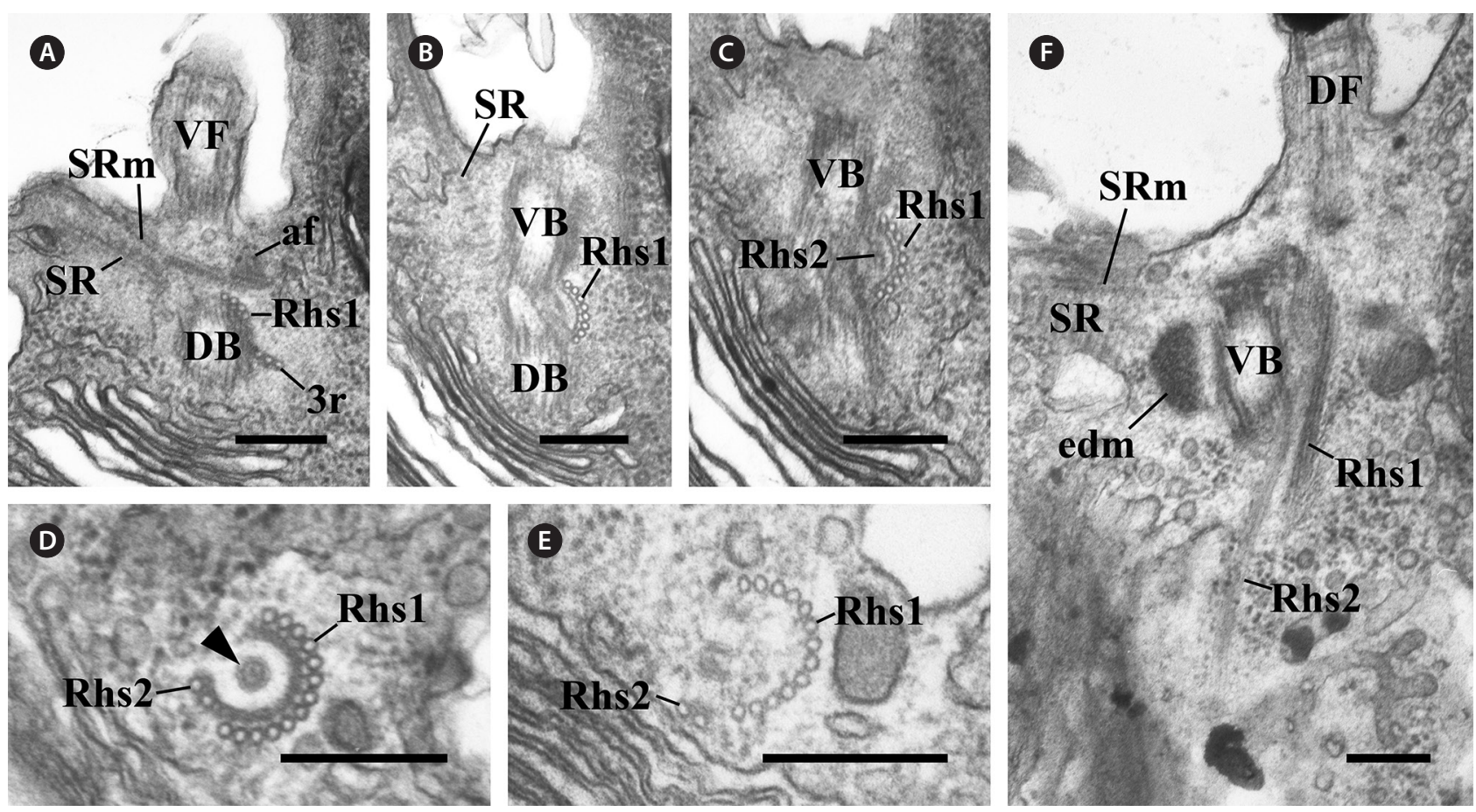

Fig. 2. Transmission electron micrographs of the rhizostyle (Rhs). (A-C) Oblique serial sections of the two basal bodies showing the Rhs1, which originates near the dorsal basal body (DB), moves toward the ventral basal body (VB) and reinforces gradually, and the Rhs2 originates on the concave surface of the Rhs1-connected electron-dense sheet. (D \& E) Cross serial sections of the Rhs showing that the Rhs 1 consists of thirteen microtubules and Rhs2 has two microtubules. The electron-dense circle can be observed (arrowhead). (F) Longitudinal section showing the two Rhs extending toward the posterior and the ventral side of the cell. af, anchoring fiber; DF, dorsal flagellum; edm, electron dense material; SR, striated fibrous root; SRm, striated fibrous root-associated microtubular root; VF, ventral flagellum; 3 r, three-stranded microtubular root. Scale bars represent: A-F, $0.2 \mu \mathrm{m}$.

\section{General ultrastructure}

Most of the organelles of C. curvata Sajeom041611A are visible in Fig. 1. The Golgi bodies were positioned at the anterior part of the cell (Fig. 1C). A chloroplast was parietally positioned under the periplast (Fig. 1E). Each pyrenoid was surrounded by starch grains (Fig. 1D \& F). The nucleomorph was located in the periplastidial compartment and was not associated with the pyrenoid (Fig. 1F). Large ejectisomes were observed near the gullet (Fig. 1C), and small ejectisomes were located at the cell periphery (Fig. 1C \& E). The nucleus was positioned at the posterior of the cell (Fig. 1C \& D).

\section{Ultrastructure of the flagellar apparatus}

The flagellar apparatus of $C$. curvata consisted of the following six major components: an Rhs; an SR; a striated fiber-associated microtubular root (SRm); an ML; a threestranded microtubular root (3r) and a two-stranded microtubular root (2r).

In the flagellar apparatus of C. curvata, the most prom- inent and conspicuous component of the flagellar apparatus was the slightly complex Rhs. The Rhs extended shortly toward the posterior of the cell (Fig. 2) and was composed of two microtubular bands (Rhs1 and Rhs2) (Table 1). Rhs1 originated from the right side of the two basal bodies and near the dorsal basal body, where Rhs1 overlapped with the $3 \mathrm{r}$ (Fig. 2A). In the cross section at the origin point, Rhs1 was composed of four microtubules without a wing-like lamellar projection (Fig. 2A). The number of microtubules in Rhsl increased rapidly with the distance from the point of origin. An electrondense sheet at the Rhsl's concave surface and wing-like connections between the electron-dense sheet and rhizostylar microtubules emerged when the number of microtubules in Rhs1 increased to eight (Fig. 2B). The electron-dense sheet and the wing-like connections were maintained until the number of microtubules of Rhs1 reached 13 (Fig. 2B-E). When the number of rhizostylar microtubules reached 13, an electron-dense circle was observed on the concave surface of the electron-dense sheet (Fig. 2D, arrowhead). As the 13 rhizostylar microtubules extended to the posteriad, the electron-dense 

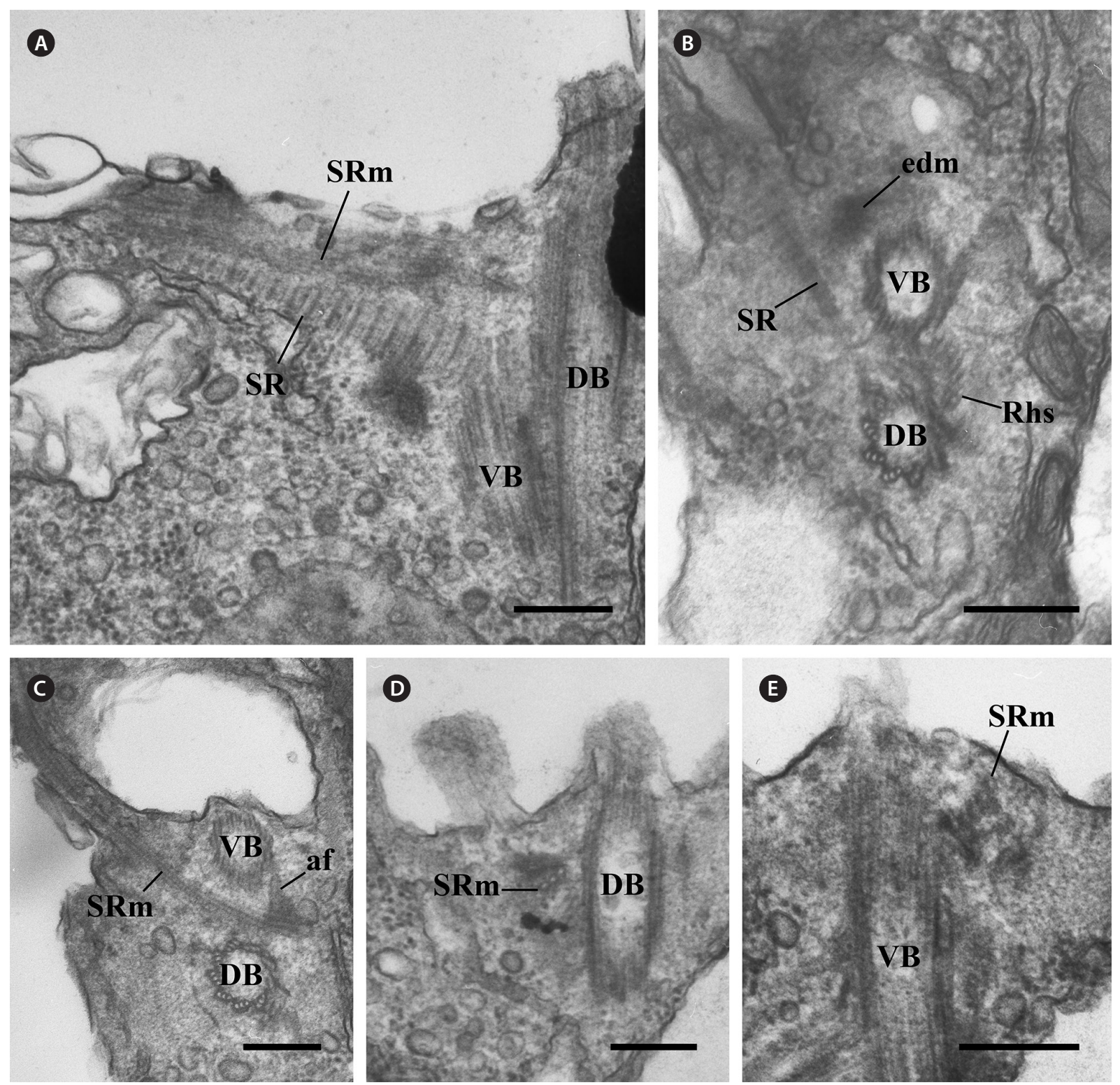

Fig. 3. Transmission electron micrographs of the striated fibrous root (SR) and the striated fibrous root-associated microtubular root (SRm). (A) Oblique section of the two basal bodies showing that the SR and SRm extend parallel to the left of the cell. (B \& C) Cross serial sections of the two basal bodies showing that the SR originates at the dorsal basal body (DB) whereas the SRm is from the right side of the two basal bodies and connects to the ventral basal body (VB) by an anchoring fiber (af). (D \& E) Serial sections showing that SRm consists of three microtubules and that the number of microtubules quickly increases to five. edm, electron-dense material; Rhs, rhizostyle. Scale bars represent: $A-E, 0.2 \mu m$.

sheet and the wing-like connection disappeared gradually (Fig. 2E); therefore, these associates existed only at the proximal region of the Rhs. Rhs2 was composed of two microtubules and originated on a concave surface of the electron-dense sheet when the number of Rhs1 microtubules reached nine (Fig. 2C). As the Rhs extended toward the postriad, Rhs2 moved toward a more dorsal side compared to Rhsl. Therefore, the distance between Rhsl and Rhs2 gradually increased.
The other major components of the flagellar apparatus were the SR and the SRm. The SR had a striped pattern with a periodicity of 37.1-45.7 nm (Table 1, Fig. 3A \& B). This fibrous root extended to the left and ventral sides of the two basal bodies with the SRm (Fig. 3A-C). The SRm was parallel with the $S R$ and originated from the right side of the two basal bodies. At the origin point of the SRm, the SRm was connected to the ventral basal body by a distinctive fibrous structure (anchoring fiber, af) that originated 


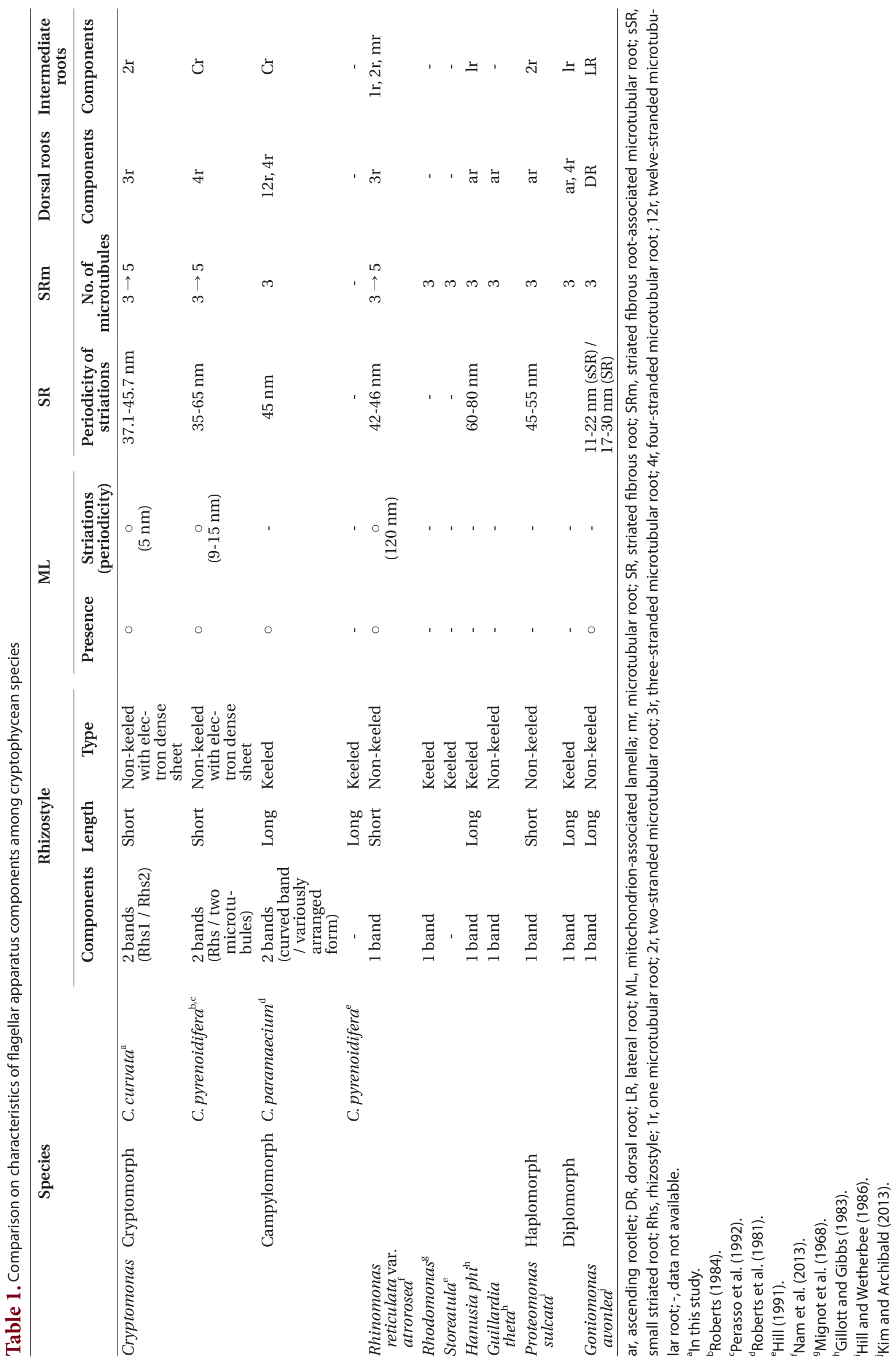




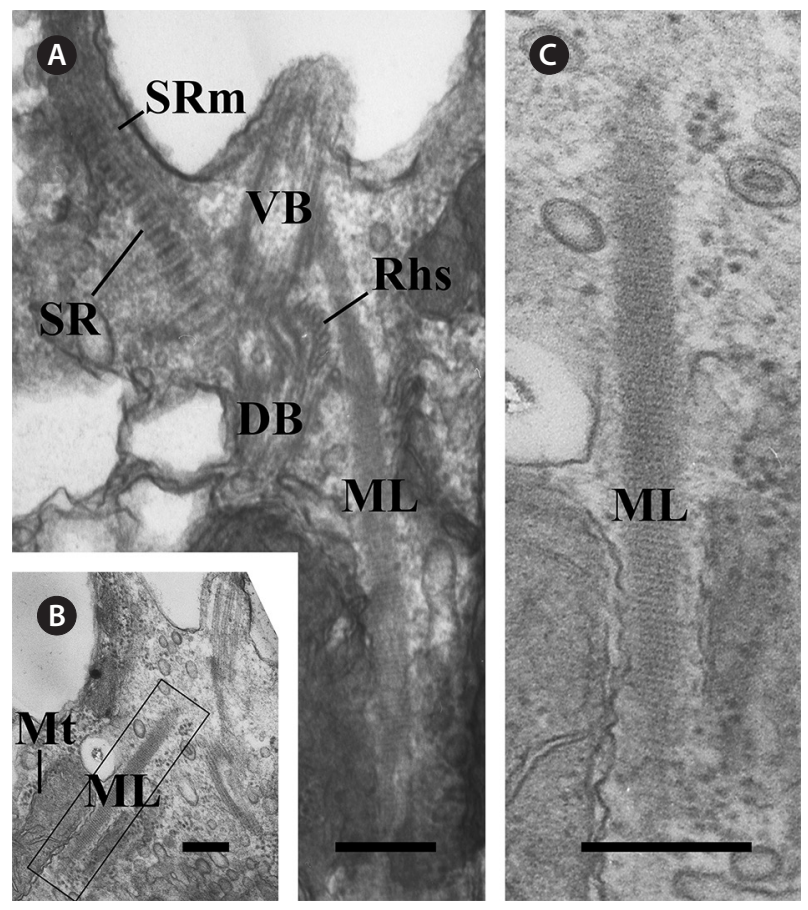

Fig. 4. Transmission electron micrographs of the mitochondrionassociated lamella (ML). (A) Oblique section of the two basal bodies showing the $M L$, which originates at the ventral basal body (VB). (B) Longitudinal section showing the ML associated with the mitochondria. (C) Enlargement of the region outlined in Figure. $B$ showing that the ML has a striated pattern. DB, dorsal basal body; Mt, mitochondria; Rhs, rhizostyle; SR, striated fibrous root; SRm, striated fibrous root-associated microtubular root. Scale bars represent: A-C, $0.2 \mu \mathrm{m}$.

at the ventral basal body (Fig. 3C). In the longitudinal serial section of the two basal bodies, the SRm comprised three microtubules near the origin point (Fig. 3D) and quickly became five (Table 1, Fig. 3E).

There was another major fibrous root among the components of the flagellar apparatus. The ML originated from the ventral basal body and extended to the posteriad of the cell (Fig. 4A). The ML was associated with the mitochondria (Fig. 4B). The ML had a striped pattern like the SR, and the periodicity of the ML was approximately 5 nm (Table 1, Fig. 4C).

In the flagellar apparatus of C. curvata, the microtubular roots, except in Rhs and SRm, were composed of two types. The first was the $3 \mathrm{r}$, which originated from the right side of the dorsal basal body (Fig. 5A). Serial sections revealed that one microtubule was added to the $3 \mathrm{r}$ immediately after its origin (Fig. 5A \& B). The $3 r$ extended in the opposite direction of the SR and SRm in the proximal region (Fig. 5C). This root added one more microtubule as it extended to the anterior of the cell near the dorsal flagellum (Fig. 5C-E). The second was the 2r, which originated
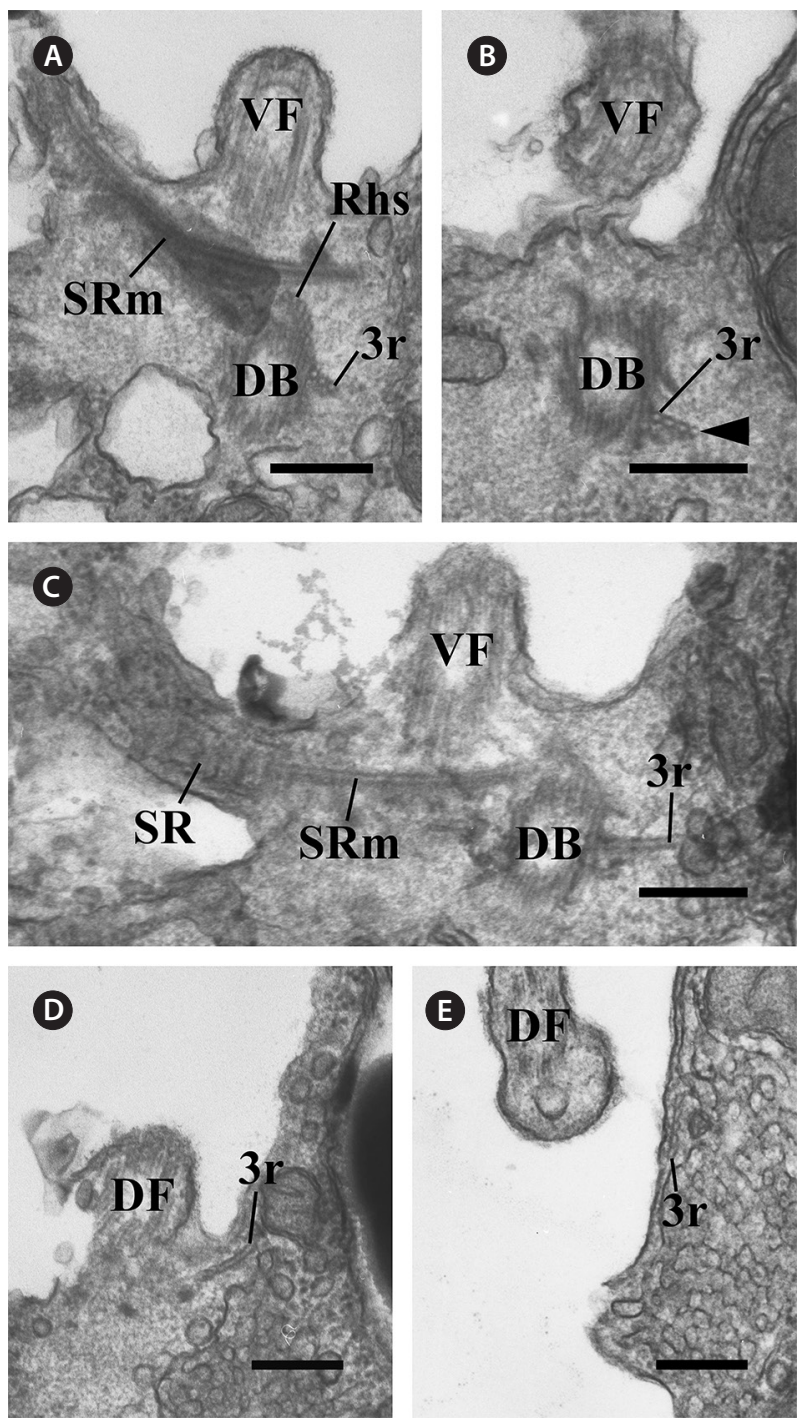

Fig. 5. Transmission electron micrographs of the three-stranded microtubular root (3r). (A \& B) Serial oblique sections of the two basal bodies showing that the $3 \mathrm{r}$ originates from the right side of the dorsal basal body (DB) and expands by one microtubule immediately (arrowhead). (C-E) Serial sections showing the $3 r$ extending toward the right side and the anteriod of the cell. DF, dorsal flagellum; Rhs, rhizostyle; SR, striated fibrous root; SRm, striated fibrous rootassociated microtubular root; VF, ventral flagellum. Scale bars represent: A-E, $0.2 \mu \mathrm{m}$.

between the two basal bodies (Fig. 6A). The 2r extended to the anterior of the cell (Fig. 6A-D) and the left side of the two basal bodies (Fig. 6E \& F). The 2r overlapped with the proximal region of Rhs1 and the SRm at a more anterior part (Fig. 6A-C).

In addition to the six major components of the flagellar apparatus mentioned above, three accessory components were observed. One fibrous connection (C) was observed between the two basal bodies (Fig. 6A). Elec- 

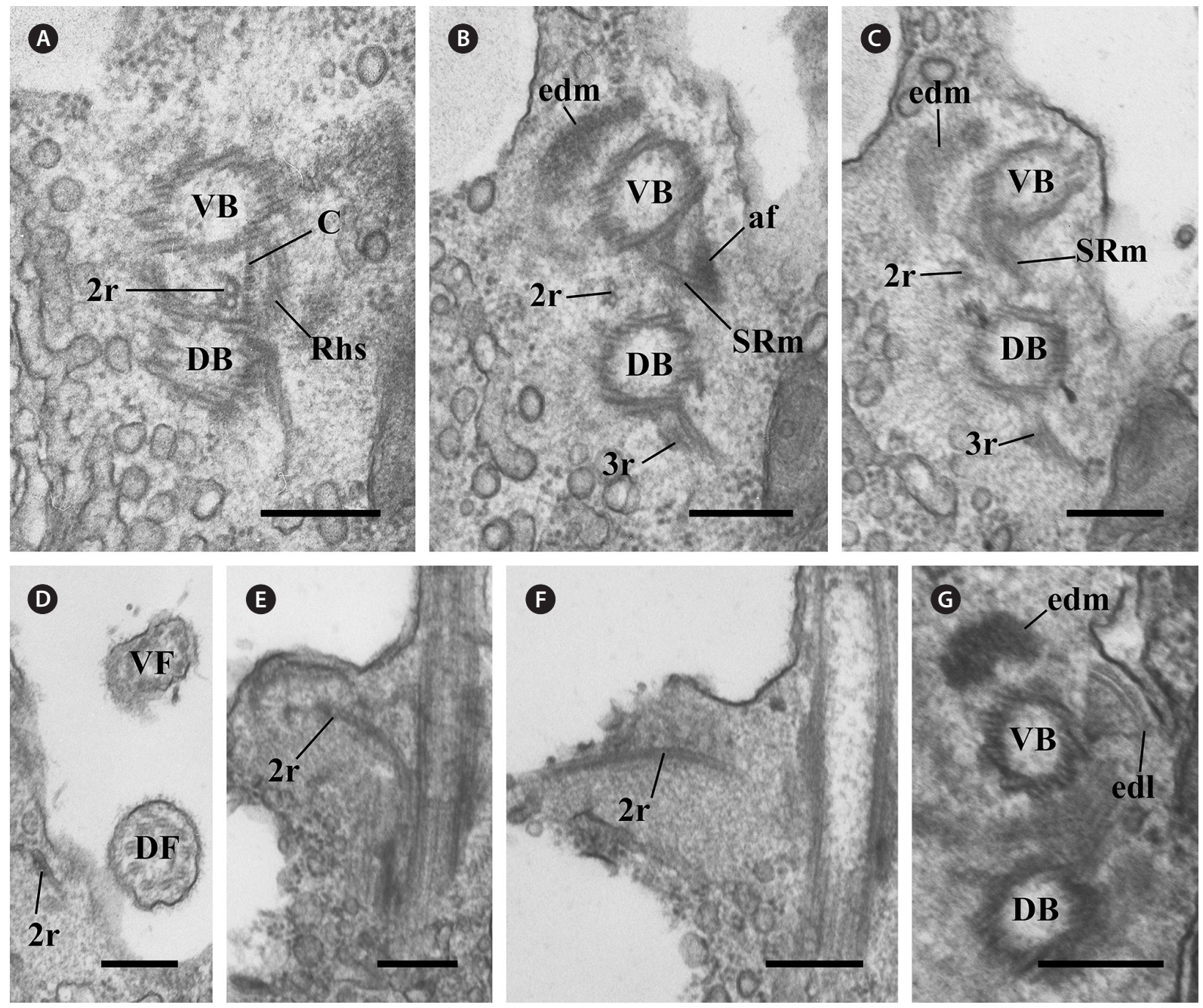

Fig. 6. Transmission electron micrographs of the two-stranded microtubular root (2r) and additional structures. (A-D) Serial sections of the basal bodies showing that the $2 r$ originates between the two basal bodies and extends to the left side of the basal bodies. The connecting structure (C) between the two basal bodies. (E \& F) Serial sections showing the $2 r$ extending shortly. (G) Cross section of the two basal bodies showing that the electron-dense layer (edl) is connected to a triplet of ventral basal bodies and the electron-dense material (edm) is associated with the ventral basal body (VB). af, anchoring fiber; DB, dorsal basal body; DF, dorsal flagellum; Rhs, rhizostyle; SRm, striated fibrous root-associated microtubular root; $V F$, ventral flagellum; 3r, three-stranded microtubular root. Scale bars represent: A-G, $0.2 \mu \mathrm{m}$.

tron-dense material was located at the ventral side of the ventral basal body, and the electron-dense layer was connected to a triplet of the ventral basal body (Fig. 6G). The diagrammatic reconstruction of $C$. curvata is intended to provide an accurate reconstruction of the flagellar apparatus but is not to scale (Fig. 7A-C).

\section{DISCUSSION}

\section{Dimorphism of the genus Cryptomonas}

Hoef-Emden and Melkonian (2003) first revealed the dimorphism (cryptomorph vs. campylomorph) of Cryptomonas species by analyzing morphological characteristics by light and electron microscopy. In spite of these morphological differences, the molecular phylogeny, based on nuclear and nucleomorph ribosomal gene sequence data of 73 strains of the genus Cryptomonas, revealed that some taxa were placed in the same lineages. Therefore, they revised the taxonomy of the genus Cryptomonas and synonymized the genera Campylomonas and Chilomonas as the genus Cryptomonas. According to these researchers, Cryptomonas ovata var. palustris UTEX 358 studied by Roberts (1984) and Perasso et al. (1992) was a cryptomorph of C. pyrenoidifera, and Campylomo- 

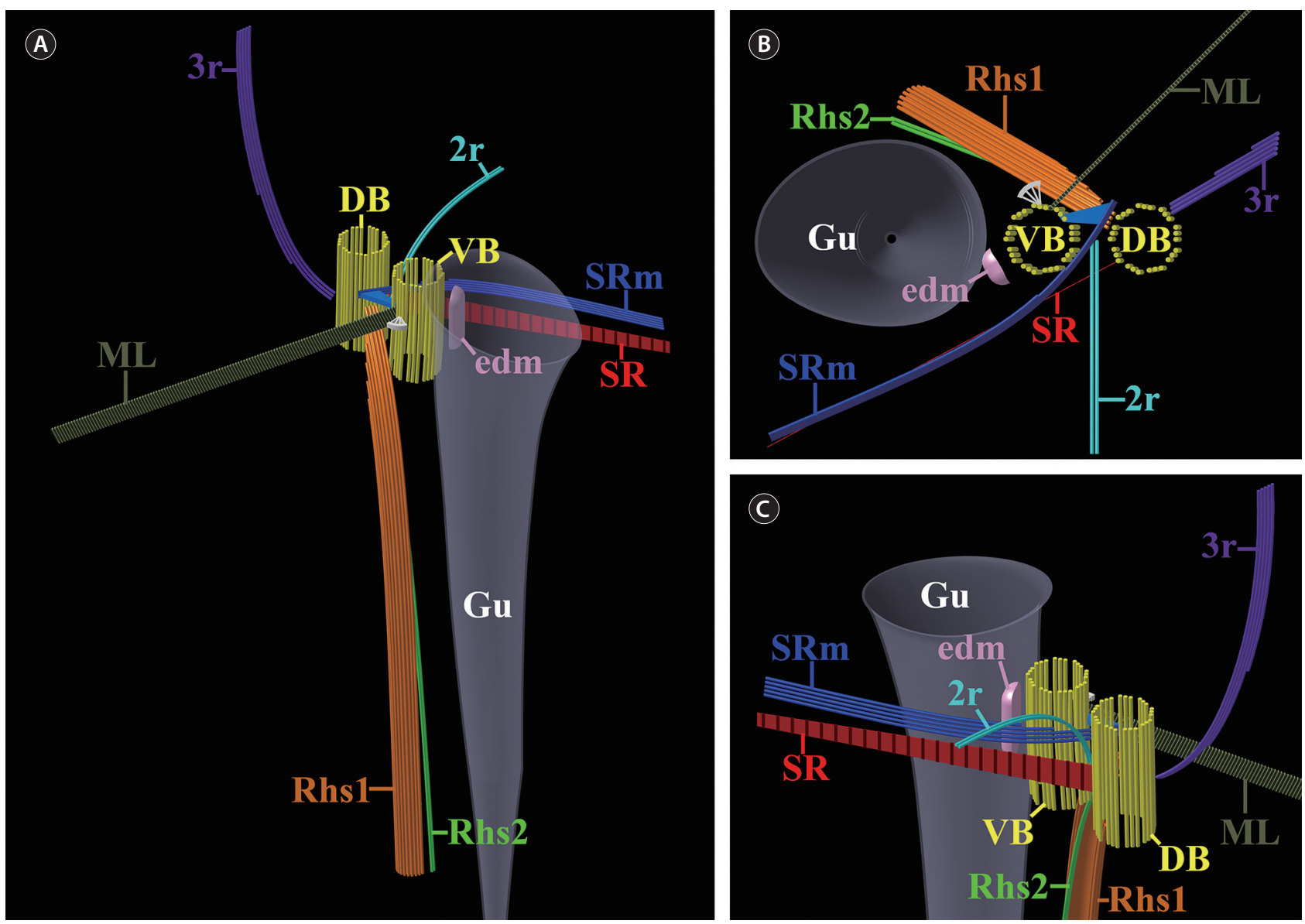

Fig. 7. Diagrammatic reconstructions of the flagellar apparatus in Cryptomonas curvata. Not to scale. (A) Diagram showing the overall flagellar apparatus. (B) Diagram showing a plane view from above. (C) Diagram showing a magnified view from the oblique left side. DB, dorsal basal body; edm, electron-dense material; Gu, gullet; ML, mitochondrion-associated lamella; Rhs1, rhizostyle1; Rhs2, rhizostyle2; SR, striated fibrous root; SRm, striated fibrous root-associated microtubular root; VB, ventral basal body; 2 r, two-stranded microtubular root; $3 r$, three-stranded microtubular root.

nas reflexa of Hill (1991) was also a campylomorph of $C$. pyrenoidifera. In addition, Chilomonas paramaecium was considered a campylomorph of C. paramaecium. In this study, we regard the C. curvata Sajeom041611A strain as a cryptomorph type based on the number of pyrenoids and the cell size under the light microscopy and TEM observations.

\section{Comparison of the flagellar apparatus in crypto- monads}

The Rhs in the cryptomorph of Cryptomonas curvata was distinctive and complex. In general, the Rhs consists of one or two bands of microtubules. The Rhs of the cryptomorph of C. curvata and C. pyrenoidifera and the campylomorph of $C$. paramaecium consists of two types of microtubular bands near the basal bodies (Roberts et al. 1981, Roberts 1984, Perasso et al. 1992) (Table 1). Additionally, the Rhs in the cryptomorph of C. curvata and C. pyrenoidifera exhibit similarities compared to other species (Table 1). First, each rhizostylar microtubule of Rhs1 in the cryptomorph of C. curvata and the Rhs in the cryptomorph of C. pyrenoidifera has a wing-like projection connecting the microtubule to the electron-dense sheet at the proximal region near the basal bodies. The wing-like projections of the Rhs in the campylomorph of C. paramaecium, in the campylomorph of C. pyrenoidifera (Hill 1991), and previously reported species with a keeled Rhs in other genera, such as $H$. phi (Gillott and Gibbs 1983) and the diplomorph of P. sulcata (Hill and Wetherbee 1986), are long and / or short in length without an electron-dense sheet. However, the cryptomorph of $C$. curvata has a short wing-like connection with the electron-dense sheet like the cryptomorph of C. pyrenoid- 
ifera. Additionally, the electron-dense sheet in the cryptomorph of C. curvata is similar to that of the cryptomorph of $C$. pyrenoidifera, including a short Rhs. A short Rhs was reported in the cryptomorph of $C$. pyrenoidifera (Roberts 1984, Perasso et al. 1992), Urgorri complanatus (LazaMartínez 2012), and $R$. reticulata var. atrorosea (Nam et al. 2013), whereas the campylomorphs of C. paramaecium (Roberts et al. 1981) and C. pyrenoidifera (Hill 1991) have a long Rhs. Therefore, the Rhs in the cryptomorph of C. curvata is quite similar to that of the cryptomorph of C. pyrenoidifera.

The other components of the flagellar apparatus were the SR and SRm. These structures always co-exist at parallel positions and exhibit some variations. One of the morphological variations is the striation periodicity of the SR (Table 1). The periodicity of the SR striped pattern in the cryptomorph of C. curvata was $37.1-45.7 \mathrm{~nm}$, similar to those of the campylomorph of C. paramaecium $(45 \mathrm{~nm})$ (Roberts et al. 1981) and the cryptomorph of $C$. pyrenoidifera (35-65 nm) (Roberts 1984). The second variation was the change in microtubule number in the SRm. In the cryptomorph of C. curvata, the number of SRm microtubules was three at its origin point and increased to five. This change in microtubule number was reported in the cryptomorph of C. pyrenoidifera (Roberts 1984), Goniomonas avonlea (Kim and Archibald 2013) and $R$. reticulata var. atrorosea (Nam et al. 2013), which have SRms composed of three microtubules and expanding up to four or five. The SRm of C. curvata is connected to the ventral basal body by an unusual striated fibrous structure called an af. The af is a distinctive structure that has not been reported in other species. However, $C$. paramaecium has a similar anchoring structure, which is a distinctive branched structure connecting the ventral basal body to the SR (Roberts et al. 1981).

The third component of the flagellar apparatus in $C$. curvata was the ML. The ML has been reported in the campylomorph of C. paramaecium (Roberts et al. 1981), the cryptomorph of C. pyrenoidifera (Roberts 1984), $R$. reticulata var. atrorosea (Nam et al. 2013) and Goniomonas avonlea (Kim and Archibald 2013). Fine striations in the ML region were observed in the cryptomorph of $C$. pyrenoidifera, $R$. reticulata var. atrorosea, and C. curvata. However, the periodicity of the ML striations of R. reticulata var. atrorosea is $120 \mathrm{~nm}$, much longer than that of the cryptomorph of C. curvata $(5 \mathrm{~nm})$ and the cryptomorph of $C$. pyrenoidifera $(9-15 \mathrm{~nm})$. The ML of $R$. reticulata var. atrorosea is divided into two directions, and the ML of the campylomorph of $C$. paramaecium consists of two layers (a thin, dense layer and a thicker, more lightly stained layer). In addition, the ML of G. avonlea has a granular appearance. Therefore, the ML of the cryptomorph of $C$. curvata is similar to that of the cryptomorph of C. pyrenoidifera with respect to the shorter periodicity of the striation and the single-layered structure.

In cryptomonad species, the number of microtubular roots differs, and each microtubular root consists of different numbers of microtubules. Nam et al. (2013) described a homologous microtubular root in previous well-studied species. According to these researchers, the microtubular roots, with the exception of the Rhs and the SRm, are classified as two types: the dorsal roots and the intermediate roots. The dorsal roots originate from the right side of the dorsal basal body and extend through a counterclockwise path. By contrast, the intermediate roots originate between the two basal bodies. In the cryptomorph of $C$. curvata, there are two types of microtubular roots: the $3 \mathrm{r}$ and the $2 \mathrm{r}$. The $3 \mathrm{r}$ originates from the right side of the dorsal basal body, and therefore the $3 \mathrm{r}$ is the only dorsal root in C. curvata. Because the $2 \mathrm{r}$ originates from between the two basal bodies, this root is an intermediate root. Specifically, the $2 \mathrm{r}$ in the cryptomorph of $C$. curvata is similar to the $\mathrm{Cr}$ root in the campylomorph of $C$. paramaecium and the cryptomorph of C. pyrenoidifera. Although P. sulcata and $H$. phi have homologous lateral rootlets (Gillott and Gibbs 1983, Hill and Wetherbee 1986), these roots do not consist of two microtubules. Therefore, the $2 \mathrm{r}$ in the cryptomorph of C. curvata and the $\mathrm{Cr}$ root in the campylomorph of C. paramaecium and the cryptomorph of C. pyrenoidifera are homologous intermediate roots, and unique characteristic features of the genus Cryptomonas.

\section{Taxonomic implication of the flagellar apparatus}

The flagellar apparatuses of seven phototrophic and a phagotrophic cryptophycean species have been described, and in the genus Cryptomonas, complete ultrastructural data and three-dimensional reconstructions of the flagellar apparatus are available for three species, including the cryptomorph of C. curvata. The reconstructed structure of the flagellar apparatus in the cryptomorph of C. curvata is more similar to those of the campylomorph of $C$. paramaecium and the cryptomorph of C. pyrenoidifera than those of H. phi, G. theta, P. sulcata, and Rhinomonas reticulate var. atrorosea due to the presence of two microtubular components in the Rhs, the striation periodicity of the SR and the ML, and the twostranded microtubular root. These characteristic features suggest that characteristics of the flagellar apparatus are conserved at the genus level in Cryptophyceae. The fea- 
tures of the Rhs are particularly useful for distinguishing closely related genera. For example, the genus Hanusia is very closely related to the genus Guillardia, as revealed in molecular phylogenetic studies (Marin et al. 1998, Deane et al. 2002, Hoef-Emden et al. 2002, Phipps et al. 2008, Tanifuji et al. 2010, Laza-Martínez 2012), and shares molecular and karyotypic features with Guillardia (Deane et al. 1998). However, Hanusia has a keeled Rhs, whereas Guillardia has a non-keeled Rhs. Thus, these two genera have slightly different flagellar root systems (Gillott and Gibbs 1983, Hoef-Emden et al. 2002). In Pyrenomonadaceae, Storeatula has a long and keeled Rhs, and Rhodomonas has a keeled Rhs, whereas Rhinomonas has a short and non-keeled Rhs (Mignot et al. 1968, Hill 1991, Nam et al. 2013).

The cryptomorphs of C. curvata and C. pyrenoidifera share common features of the flagellar apparatus, such as the short and non-keeled Rhs, rhizostylar microtubules connected to the electron-dense sheet by wing-like projections, and the periodicity of the ML striations. The campylomorph of $C$. pyrenoidifera is closely related to that of C. paramaecium in terms of its long and keeled Rhs. P. sulcata has two morphologically distinct life histories, the haplomorph and diplomorph, which have the same features of furrow and gullet and the same type of biliprotein but differ in their flagellar apparatus (longkeeled Rhs in the diplomorph vs. shorter non-keeled Rhs in the haplomorph). Thus, features of the flagellar apparatus may differ in the dimorphism of the cryptomonad species. According to Hoef-Emden and Melkonian (2003) and Hoef-Emden (2007), molecular data indicate that $C$. curvata has a closer phylogenetic relationship with C. pyrenoidifera than C. paramaecium. Although available ultrastructural data for the flagellar apparatus in the genus Cryptomonas are limited, the whole structural similarity of the flagellar apparatus in the genus Cryptomonas is congruent with the molecular phylogenetic data (Choi et al. 2013). Thus, the ultrastructure of the flagellar apparatus may have useful diagnostic features to infer phylogenetic relationships at the interspecies level.

\section{ACKNOWLEDGEMENTS}

This work was supported by the 2014 CNU research fund of Chungnam National University and the Basic Core Technology Development Program for the Oceans and the Polar Regions of the National Research Foundation (NRF) funded by the Ministry of Science, ICT \& Future Planning (NRF-2015M1A5A1041808).

\section{REFERENCES}

Choi, B., Son, M., Kim, J. I. \& Shin, W. 2013. Taxonomy and phylogeny of the genus Cryptomonas (Cryptophyceae, Cryptophyta) from Korea. Algae 28:307-330.

Clay, B. L., Kugrens, P. \& Lee, R. E. 1999. A revised classification of Cryptophyta. Bot. J. Linn. Soc. 131:131-151.

Deane, J. A., Hill, D. R. A., Brett, S. J. \& McFadden, G. I. 1998. Hanusia phi gen. et sp. nov. (Cryptophyceae): characterization of 'Cryptomonas sp. Ф'. Eur. J. Phycol. 33:149-154.

Deane, J. A., Strachan, I. M., Saunders, G. W., Hill, D. R. A. \& McFadden, G. I. 2002. Cryptomonad evolution: nuclear 18S rDNA phylogeny versus cell morphology and pigmentation. J. Phycol. 38:1236-1244.

Gillott, M. A. \& Gibbs, S. P. 1983. Comparison of the flagellar rootlets and periplast in two marine cryptomonads. Can. J. Bot. 61:1964-1978.

Guiry, M. D. \& Guiry, G. M. 2015. AlgaeBase. World-Wide Electronic Publication. National University of Ireland, Galway. Available from: http://www.algaebase.org. Accessed Sep 10, 2015.

Hill, D. R. A. 1991. A revised circumscription of Cryptomonas (Cryptophyceae) based on examination of Australian strains. Phycologia 30:170-188.

Hill, D. R. A. \& Rowan, K. S. 1989. The biliproteins of the Cryptophyceae. Phycologia 28:455-463.

Hill, D. R. A. \& Wetherbee, R. 1986. Proteomonas sulcata gen. et sp. nov. (Cryptophyceae) a cryptomonad with two morphologically distinct and alternating forms. Phycologia 25:521-543.

Hoef-Emden, K. 2007. Revision of the genus Cryptomonas (Cryptophyceae) II: incongruences between the classical morphospecies concept and molecular phylogeny in smaller pyrenoid-less cells. Phycologia 46:402-428.

Hoef-Emden, K., Marin, B. \& Melkonian, M. 2002. Nuclear and nucleomorph SSU rDNA phylogeny in the Cryptophyta and the evolution of cryptophyte diversity. J. Mol. Evol. 55:161-179.

Hoef-Emden, K. \& Melkonian, M. 2003. Revision of the genus Cryptomonas (Cryptophyceae): a combination of molecular phylogeny and morphology provides insights into a long-hidden dimorphism. Protist 154:371-409.

Kim, E. \& Archibald, J. M. 2013. Ultrastructure and molecular phylogeny of the cryptomonad Goniomonas avonlea sp. nov. Protist 164:160-182.

Laza-Martínez, A. 2012. Urgorri complanatus gen. et sp. nov. (Cryptophyceae), a red-tide-forming species in brackish waters. J. Phycol. 48:423-435.

Marin, B., Klingberg, M. \& Melkonian, M. 1998. Phylogenetic relationships among the Cryptophyta: analyses 
of nuclear-encoded SSU rRNA sequences support the monophyly of extant plastid-containing lineages. Protist 149:265-276.

Mignot, J. -P., Joyon, L. \& Pringsheim, E. -G. 1968. Compléments a l'étude cytologique des Cryptomonadines. Protistologica 4:493-506.

Nam, S. W., Go, D., Son, M. \& Shin, W. 2013. Ultrastructure of the flagellar apparatus in Rhinomonas reticulata var. atrorosea (Cryptophyceae, Cryptophyta). Algae 28:331341.

Nam, S. W., Shin, W., Coats, D. W., Park, J. W. \& Yih, W. 2012. Ultrastructure of the oral apparatus of Mesodinium rubrum from Korea. J. Eukaryot. Microbiol. 59:625-636.

Novarino, G. 2003. A companion to the identification of cryptomonad flagellates (Cryptophyceae $=$ Cryptomonadea). Hydrobiologia 502:225-270.

Perasso, L., Hill, D. R. A. \& Wetherbee, R. 1992. Transformation and development of the flagellar apparatus of Cryptomonas ovata (Cryptophyceae) during cell division. Protoplasma 170:53-67.
Phipps, K. D., Donaher, N. A., Lane, C. E. \& Archibald, J. M. 2008. Nucleomorph karyotype diversity in the freshwater cryptophyte genus Cryptomonas. J. Phycol. 44:11-14. Reynolds, E. S. 1963. The use of lead citrate at high $\mathrm{pH}$ as an electron-opaque stain in electron microscopy. J. Cell Biol. 17:208-212.

Roberts, K. R. 1984. Structure and significance of the cryptomonad flagellar apparatus. I. Cryptomonas ovata (Cryptophyta). J. Phycol. 20:590-599.

Roberts, K. R., Stewart, K. D. \& Mattox, K. R. 1981. The flagellar apparatus of Chilomonas paramecium (Cryptophyceae) and its comparison with certain zooflagellates. J. Phycol. 17:159-167.

Tanifuji, G., Onodera, N. T. \& Hara, Y. 2010. Nucleomorph genome diversity and its phylogenetic implications in cryptomonad algae. Phycol. Res. 58:230-237.

Xia, S., Liu, G. -X. \& Hu, Z. -Y. 2013. Morphological examination and phylogenetic position of two newly recorded freshwater Cryptomonas species (Cryptophyceae) from China. J. Syst. Evol. 51:212-222. 